\title{
EEG SEIZURE DETECTION BY INTEGRATING SLANTLET TRANSFORM WITH SPARSE CODING
}

\author{
Ali K Ibrahim, Hanqi Zhuang, Nurgun Erdol, and Ali Muhmed Ali \\ Department of Computer and Electrical Engineering and Computer Science \\ Florida Atlantic University \\ Boca Raton, FL 33431 \\ Email: \{aibrahim, zhuang, erdol, aibrahim2014\}@fau.edu
}

\begin{abstract}
EEG signals, recording both normal and abnormal activities of neurons in the brain, are widely used in epilepsy detection. In this paper, an EEG signal classification method based on Slantlet Transform and sparse coding is proposed to greatly reduce number of false alarms and improve speed of detection. With Slantlet Transform, salient information of EEG signals is mapped into a sparse space. In order to ensure good detection rates from the EEG signals, a Sparse Representation Classifier is adopted. Experimental results demonstrate that the in terms of detection rate, the proposed method, which integrates Slantlet Transform with sparse coding, outperforms the current state-of-the-art EEG seizure detection methods.
\end{abstract}

Index Terms - EEG signal,seizure, Slantlet Transform, Sparse Representation Classifier.

\section{INTRODUCTION}

Epilepsy is a chronic neurological brain disorder that affects around 50 million people of all ages in the world. Recurrent seizures are the distinct symptoms in epilepsy according to the World Health Organization (WHO). Seizures are physical reactions to sudden, usually brief, excessive electrical discharges in a group of brain cells [1]. Empirical analysis and monitoring of this disease depicts two unique types of epilepsy; behavioral and electrographic. Epilepsy can manifest itself in a behavioral seizure in which the patient himself can experience the episode, can be seen by an observer or recorded on video. An electrographic (or electroencephalographic (EEG)) seizure is defined as an abnormal paroxysmal EEG pattern. In many cases, there is a strong correlation between behavior seizure and EEG signals [2].

Since the 1970s, research and advancements in detecting automatic EEG seizures, quantifying and recognizing them continued in the medical, physical and engineering fields. In one of the earliest studies, Gotman [3] presented an automated seizure detection method based on halfwave decomposition of EEG signals. This method was evaluated on a scalp EEG dataset obtained from 16 pa- tients and intracranial EEG recorded from 4 patients.76\% detection accuracy were reported in addition to a false detection rate of 1 per hour. Later, Gabor et. al. [4] proposed a method to detect epileptic seizures using wavelets and self-organizing neural networks. From a pool of 22 patients, 24 long term scalp EEG readings were obtained and the aforementioned algorithm was applied. 90\% detection accuracy was reported with a mean false detection rate of 0.71 per hour. Even though these early studies emphasized requirement of a more robust methods, they identified the main characteristics of seizure EEG as selfsimilar oscillatory patterns. Polychronaki et al. [5] have performed a comparison of FD estimation algorithms for epileptic seizure detection. The authors show that Knearest neighbor offers the best seizure detection performance. In their research, they have concluded that the knearest neighbors algorithm provides the best performance metrics. Robust seizure detection however, can't be sustained by depending on FD estimation algorithms due to latency inherently present in seizure detection and false positive rates associated with FD computation. Multivariate analysis of EEG effectiveness in capturing the changes in spatial domain was studied and discussed by Mierlo et al [6]. These changes in spatial domains are indicative of a seizure onset and can be utilized for early detection. Moreover, the onset of seizure causes an increase in the spatial connectivity patterns in the brain which might benefit seizure early detection [7]. Capturing those connectivity patterns, using network based approach, have led to several studies, which reviewed by Yaffe et al [8]. These studies emphasized on the importance on including these spatial and temporal patterns for better and early detection. In [9], non-linear features were used for EEG classification, resulting in an overall $96.5 \%$ accuracy.

The time-limited oscillatory features of the EEG seizures have led researchers to use various time-frequency analysis methods $[11,12,13,14]$ for detection. The oscillatory patterns of EEG seizures can be captured using wavelet based time-frequency techniques. However, these methods are still handcrafted and require users defined parameters which may not be feasible for developing a robust and efficient seizure detection algorithm. The need therefore to design a system that 'learns' intricate features of EEG seizures is desirable for more robust seizure detection. 
In this research, a sparse coding technique is applied to construct patient-specific detectors capable of detecting seizure onsets with high accuracy and high processing speed. The patient-specific detectors are evaluated using pediatric scalp EEG signals because these exhibit greater variability in seizure and nonseizure activities. A key component to the relatively high accuracy of the Sparse Representation Classifier is the use of Slantlet Transform for extracting feature vectors that are capable of converting the EEG signals to sparse features.

This paper is organized as follows. In Section II, background materials on the EGG dataset used in the experiment, the Slantlet Transform for feature extraction, and Sparse Representation Classifier are provided. Section III presents the detection results with the proposed approach, and these are compared with previous works. Concluding remarks are given in Section IV.

\section{METHODOLOGY}

\section{A. EEG Data}

The pediatric EEG data used in this paper is from the CHB-MIT database, which can be downloaded from the PhysioNet website:

http://physionet.org/physiobank/database/chbmit/.

This dataset, with 916 hours of recording, contains EEG signals from 24 patients, and each patient has his/her own recoding. Also, this dataset contains 173 test seizures, including various seizure types.

\section{B. Slantlet Transform}

The SLT, an orthogonal Discrete Wavelet Transform (DWT) with two zero moments, possesses different filters with different length for each scale [15]. With this design, it improves time localization. The parallel structure used in implementation of DWT provides more flexibility in designing filters that target different data features. A two scale orthogonal DWT having an iterated filter bank with two zero moments and its corresponding filter bank realized using SLT is shown in Fig.1.

The desirable properties of orthogonality and two vanishing moments are maintained by this SLT filter bank [15]. At each level, different filters are implemented. Moreover, the filter bank structure of Slantlet Transform depends on number of levels that being used. For an $l-$ levels SLT filter bank, it has $2 l$ channels. The first channel consists of filter $h_{l}(n)$ followed by filter $f_{l}(n)$ in the second channel. A down sampling by $2^{l}$ is preceded by both $h_{l}(n)$ and $f_{l}(n)$. The remaining $2 l-1$ channels are filtered by $g_{i}(n)$ and its shifted time reverse for $i=$ $1,2, \ldots l-1$, are then down sampled by $2^{i+1}$. The $h_{l}(n)$, $f_{l}(n)$, and $g_{i}(n)$ filters are piecewise linear, hence each filter can be represented as the sum of DC and a linear term. The general equations of $h_{l}(n), f_{l}(n)$, and $g_{i}(n)$ are given in (1):

$$
\begin{aligned}
& g_{i}(n)=\left\{\begin{array}{c}
a_{0,0}+a_{0,1} \text { for } n=0,1, \ldots .2^{i}-1 \\
a_{1,0}+a_{1,1} \text { for } n=2^{i}, \ldots .2^{i+1}-1
\end{array}\right. \\
& h_{i}(n)=\left\{\begin{array}{c}
b_{0,0}+b_{0,1} \text { for } n=0,1, \ldots .2^{i}-1 \\
b_{1,0}+b_{1,1} \text { for } n=2^{i}, \ldots .2^{i+1}-1
\end{array}\right. \\
& f_{i}(n)=\left\{\begin{array}{c}
c_{0,0}+c_{0,1} \text { for } n=0,1, \ldots .2^{i}-1 \\
c_{1,0}+c_{1,1} \text { for } n=2^{i}, \ldots .2^{i+1}-1
\end{array}\right.
\end{aligned}
$$

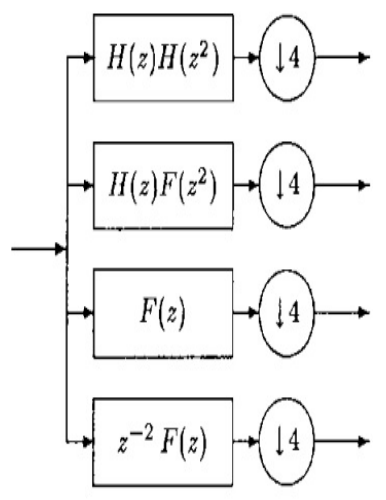

(a)

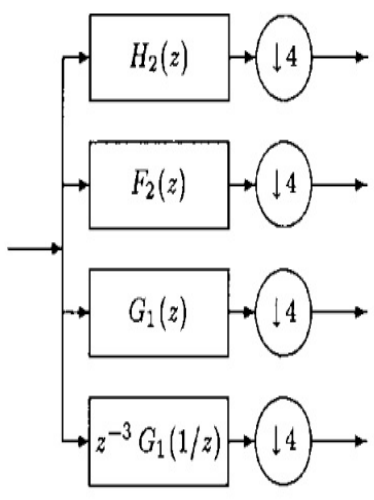

(b)
Fig.1 (a) Two-scale iterated filter bank and (b) corresponding two-scale Slantlet filter bank

The solution to (1) is solved by subjecting (1) to several constraints. All three functions need to satisfy the unit form condition and orthogonality to their shifted time reverse condition. Both $g_{i}(n)$ and $f_{i}(n)$ need to annihilate linear discrete time polynomials [15].

\section{Sparse Representation Classification}

The underline research problem has two classes, and each class has a set of $N$ features vectors extracted using Slantlet Transform. Assume $a_{i j} \in R^{d}$ a features vector extracted from $j$ th window. let $A=\left[A_{1} A_{2}\right]$,where $A_{k}=$ $\left[\boldsymbol{a}_{k 1} a_{k 2} \boldsymbol{a}_{k 3} \ldots a_{k N}\right] \in R^{d \times N}(k=1,2)$ represent training samples for class $k$. Thus, the dictionary $A$ has two class, and each class has $N$ training samples as in equation (2)

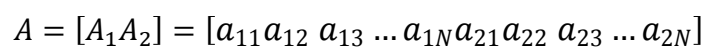

Let the measurement vector, $\boldsymbol{y} \in R^{d}$, which is extracted from a testing signal, be expressed as a linear combination of the columns of dictionary $A$ as:

$$
y=\alpha_{11} a_{11}+\alpha_{12} a_{12}+\cdots \cdot \alpha_{1 N} a_{1 N}
$$

The above equation can be written in a matrix form,

where

$$
y=A x
$$

$$
\boldsymbol{x}=\left[\begin{array}{llll}
\alpha_{11} & \alpha_{12} & \alpha_{13} & \ldots \\
\alpha_{1 N}
\end{array}\right]
$$


The problem is to find $\boldsymbol{x}$ from (3). Since the dimension of $y$ is smaller than that of $\boldsymbol{x}$ in our problem, we use the fallowing objective function to find a sparse solution of equation (3):

$$
\min _{x}\|x\|_{0} \text { subject to } \boldsymbol{y}=A \boldsymbol{x}
$$

where $\|x\|_{0}$, norm zero of $\boldsymbol{x}$, denotes the number of nonzero coefficients in $\boldsymbol{x}$. Solutions to this problem can be found in [16] and [17].

In this work, we have chosen OMP algorithm to obtain solution to (4) [18], due to its simplicity. In the OMP algorithm, columns of $A$ are selected to be the basis of $\boldsymbol{y}$. The algorithm picks those representative columns in the following manner [16]. To start with, $\boldsymbol{x}$ is initialized to $\mathbf{0}$. At each iteration, one column of $A$ is selected to be orthogonal to the residual,

$$
\boldsymbol{r}(\boldsymbol{y})=\boldsymbol{y}-A \boldsymbol{x}
$$

The corresponding coefficient of $\boldsymbol{x}$ is then updated, and procedure is repeated until the residual error bellows a preset threshold value. For a more detailed description of the algorithm, readers are referred to $[16,18]$.

\section{DETECTION RESULTS}

We characterized the performance of our seizure detector in terms of detections rate, and specificity. Specificity refers to the number of times, over the course of 24 hours, that the detector declared the onset of seizure activity in the absence of an actual clinical seizure.

Fig. 2 shows the number of false detections declared by the detector per 24 hours for each of the 24 subjects using SLT features and the SRC classifier. The median false detection rate is 2 false detections per 24 hour period.

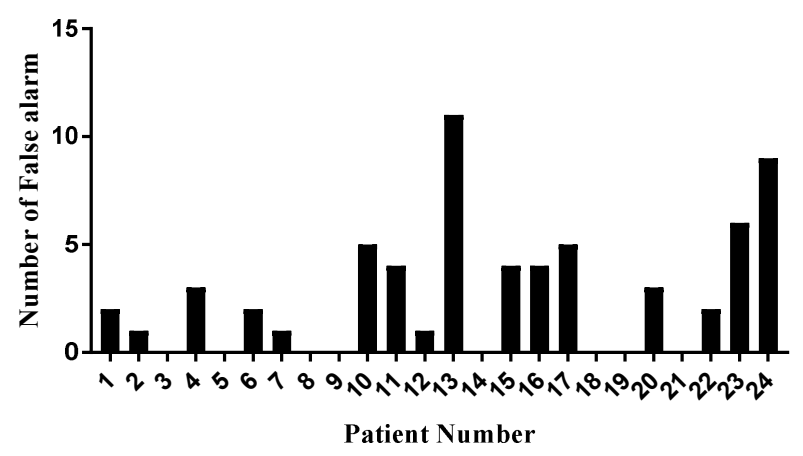

Fig. 2 Number of missing detection per patient using SLT features and SRC classifier.

In Fig. 2 we see that patient 13 has many false seizure detections, which is 11 per 24 hour period. However, the algorithm catches all seizures related to patients $3,5,8,9,14,18,19$, and 21 .
The comparison between DWT features and Slantlet features with Sparse Representation Classifier (SPR) is shown in Fig. 3. It is observed that Slantlet features has fewer miss detections of seizures than DWT features. The main reason that makes SLT work better with SPR is that SLT features are more sparse than DWT features. Therefore, SPR works better with Slantlet Transform.



Fig. 3 Comparison between SLT features and DWT features in seizures detection.

Fig. 4 compares the results obtained by SLT features and sparse classifier with detections results obtained by the method given in [19]. Marked improvements are obtained by the proposed method in terms of number of undetected seizures.



Fig.3 Comparison between SLT features and DWT features in seizures detection.

Table 1 compares the results obtained by using the two types of features (SLT and DWT) with various classifiers. It is clear that SLT+SPC produces the best seizure detection performance. Different types of window size were also tested in the experiment. We can see that the effect of reducing window size increase the accuracy of detection. 
The smallest window size chosen is $1 \mathrm{sec}$ which is 256 samples.

To compare Slantlet with DWT further, the Receiver Operating Characteristics (ROC) curves, which are the plots of true positive rate (correctly classified upcall) against false positive rate (non-upcall classified as upcall), are shown for all scenarios in Fig.4 (with the SVM as the chosen classifier).

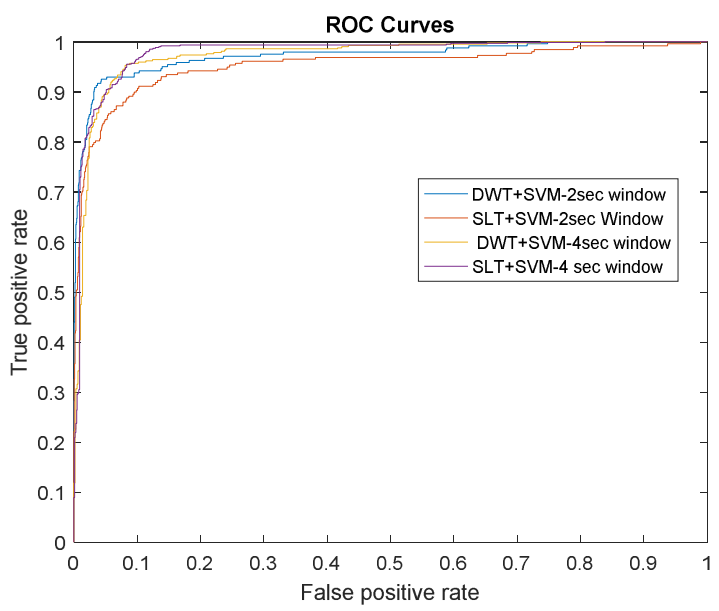

Fig. 4 ROC curves

\section{CONCLUSIONS}

We presented and evaluated a method of using Slantlet Transform as a feature extractor and Sparse Representation Classifier as a detector to detect the onset of epileptic seizures. The combination of Slantlet Transform with Sparse Representation Classifier improves the seizure detection rate, in comparison with other feature extractor and classifier combinations.

Table 1 Seizure detection results using different types of classifiers

\begin{tabular}{cccc}
\hline Method & $\begin{array}{c}\text { Window } \\
\text { length (8- } \\
\text { sec) }\end{array}$ & $\begin{array}{c}\text { Window } \\
\text { length(4- } \\
\text { sec) }\end{array}$ & $\begin{array}{c}\text { Window } \\
\text { length(1-sec) }\end{array}$ \\
\hline DWT+SVM & $84.64 \%$ & $92.62 \%$ & $96.8 \%$ \\
SLT+SVM & $86.14 \%$ & $91.38 \%$ & $96.3 \%$ \\
DWT+KNN & $81.93 \%$ & $88.43 \%$ & $93.1 \%$ \\
SLT+KNN & $83.71 \%$ & $85.91 \%$ & $93.6 \%$ \\
DWT+SPC & $87.35 \%$ & $93.4 \%$ & $97.4 \%$ \\
SLT+SPC & $89.4 \%$ & $93.8 \%$ & $98.2 \%$ \\
\hline
\end{tabular}

\section{References}

[1] World Health Orgnization, 2011. Available: http://www.who.int/medicare/?factsheets/fs999/en/.

[2] J.Gotman, "Automatic detection of seizures and spikes,"J.Clin Neurophsiol,16:130-140,1999.
[3] J.Gotman, "Automatic Recognition of eplitptic seizures in the EEG,"Electroencephalography and Clinical Neurophoysiology, vol.54,pp530-540,11//1982 .

[4] Gabor AJ, Leach RR, Dowla FU. Automated seizure detection using a self-organizing neural network. Electroencephalogr Clin Neurophysiol 1996;99:257-66.

[5] G. E. Polychronaki, P. Ktonas, S. Gatzonis, P. A. Asvestas, E. Spanou, A. Siatouni, et al., "Comparison of fractal dimension estimation algorithms for epileptic seizure onset detection," in BioInformatics and BioEngineering, 2008. BIBE 2008. 8th IEEE International Conference on, 2008, pp. 1-6.

[6] P. van Mierlo, M. Papadopoulou, E. Carrette, P. Boon, S. Vandenberghe, K. Vonck, et al., "Functional brain connectivity from EEG in epilepsy: Seizure prediction and epileptogenic focus localization," Progress in Neurobiology, vol. 121, pp. 19-35, Oct. 2014.

[7] R. Yaffe, S. Burns, J. Gale, H. J. Park, J. Bulacio, J. GonzalezMartinez, et al., "Brain state evolution during seizure and under anesthesia: A network-based analysis of stereotaxic eeg activity in drug-resistant epilepsy patients," in 2012 Annual International Conference of the IEEE Engineering in Medicine and Biology Society, 2012, pp. 5158-5161.

[8] R. B. Yaffe, P. Borger, P. Megevand, D. M. Groppe, M. A. Kramer, C. J. Chu, et al., "Physiology of functional and effective networks in epilepsy," Clinical Neurophysiology, vol. 126, pp. 227 236, Feb. 2015.

[9] Q. Yuan, W. Zhou, S. Li, and D. Cai, "Epileptic EEG classification based on extreme learning machine and nonlinear features," Epilepsy Research, vol. 96, no. 1, pp. 29-38, Sep. 2011.

[10] R. G. Andrzejak, K. Lehnertz, F. Mormann, C. Rieke, P. David, and C. E. Elger, "Indications of nonlinear deterministic and finitedimensional structures in time series of brain electrical activity: Dependence on recording region and brain state," Physical Review $E$, vol. 64, no. 6, p. 061907 , Nov. 2001

[11] M. E. Saab and J. Gotman, "A system to detect the onset of epileptic seizures in scalp EEG," Clinical Neurophysiology, vol. 116, pp. 427-442, 2// 2005.

[12] L.Kuhlmann,AN.Burkitt,M.J.Cook,K.Fuller,D.B.Grayden,L.Seider er,et al., "Seizures detection using seizure probability estimation: comparsian of featuresused detect seizures,"Annals of biomedical engineering,vol.37,pp.2129-2145,2009.

[13] A. Shoeb, H. Edwards, J. Connolly, B. Bourgeois, S. Ted Treves, and J. Guttag, "Patient-specific seizure onset detection," Epilepsy \& Behavior, vol. 5, pp. 483-498, 8// 2004.

[14] A. Shoeb, A. Kharbouch, J. Soegaard, S. Schachter, and J. Guttag,"A machine learning algorithm for detecting seizure termination in scalp EEG,"Epilpsy \& Behavior,vol.22 ,supplement 1, pp. S36-S43,12//2011.

[15] I.W. Selesnick (1999), The Slantlet transform, IEEE Trans. Signal Process., vol. 47, no. 5, pp. 1304-1313.

[16] Michael Elad, Sparse and Redundant Representations, Springer, New York, 2009.

[17] Y.C.Pati, R. Rezaiifar, and P. S. Krishnaprasad,"Orthogonal matching pursuit : : Recursive function approximation with application to wavelet decomposition,"in Proceedings of the 27th Annual Alislmor Conference on Signals, Systems, and Computers,1993,pp.40-44.

[18] Joel A.Troppand Anna C.Gilbert,"Signal recovery from random measurements via orthogonal matching pursuit,"IEEE Trans.Inform. Theory,vol.53,pp.4655-4666,2007.

A. Shoeb and J. Guttag, "Application of machine learning to epileptic seizure detection," in Proceedings of the 27th International Conference on Machine Learning (ICML-10), J. Fürnkranz and T. Joachims, Eds. Haifa, Israel: Omnipress, June 2010, pp. 975-982. 